This is an electronic reprint of the original article. This reprint may differ from the original in pagination and typographic detail.

Author(s): Juutilainen, Antti; Volodin, A.; Ahlskog, Markus

Title: $\quad$ Measurements of tunneling conduction to carbon nanotubes and its sensitivity to oxygen gas

Year: $\quad 2012$

Version:

Please cite the original version:

Juutilainen, A., Volodin, A., \& Ahlskog, M. (2012). Measurements of tunneling conduction to carbon nanotubes and its sensitivity to oxygen gas. Physical Review $B$, 86, 45405. https://doi.org/10.1103/PhysRevB.86.045405

All material supplied via JYX is protected by copyright and other intellectual property rights, and duplication or sale of all or part of any of the repository collections is not permitted, except that material may be duplicated by you for your research use or educational purposes in electronic or print form. You must obtain permission for any other use. Electronic or print copies may not be offered, whether for sale or otherwise to anyone who is not an authorised user. 


\title{
Measurements of tunneling conduction to carbon nanotubes and its sensitivity to oxygen gas
}

\author{
A. Juutilainen and M. Ahlskog* \\ ${ }^{1}$ University of Jyväskylä, Nanoscience Center, P.O.B 35 (YFL), FI-40014, Finland
}

A. Volodin

${ }^{2}$ Laboratorium voor Vaste-Stoffysica en Magnetisme, Katholieke Universiteit Leuven, B-3001 Leuven, Belgium

(Received 21 April 2012; published 5 July 2012)

\begin{abstract}
We have measured the conductive properties of junctions between carbon nanotubes (CNT) and non-noble metals $M(M=\mathrm{Al}, \mathrm{Ti}, \mathrm{Nb})$, which are separated by the native oxide $\left(M_{\mathrm{OX}}\right)$ of the metal. Reproducible and asymmetric current-voltage characteristics were obtained from $\mathrm{Ti} / \mathrm{Ti}_{\mathrm{OX}} / \mathrm{CNT}$ and $\mathrm{Nb} / \mathrm{Nb}_{\mathrm{OX}} / \mathrm{CNT}$ junctions, while $\mathrm{Al} / \mathrm{Al}_{\mathrm{OX}} / \mathrm{CNT}$ exhibited no current until breakdown, which is attributed to the larger bandgap of $\mathrm{Al}_{\mathrm{Ox}}$. The conduction in the Ti- and Nb-based junctions is not due to direct tunneling since they exhibit strong temperature dependence. The presence of oxygen is shown to drastically, but reversibly, modify the current-voltage characteristics, and in particular its asymmetry. The observed phenomena are discussed in terms of an elementary model where the different work functions of $M$ and the CNT determine the asymmetric barrier of the junction. These results are complemented with Kelvin probe microscopy measurements of the work function distribution in the vicinity of the junctions in different gas environments. Three-terminal field effect transistor devices are also demonstrated, where a $M / M_{\mathrm{OX}}$ metal electrode acts as the gate electrode.
\end{abstract}

DOI: 10.1103/PhysRevB.86.045405 PACS number(s): 73.63.Fg, 74.62.Dh, 73.40.Gk, 72.80.Rj

\section{INTRODUCTION}

Experimental work on the transport properties of carbon nanotubes $(\mathrm{CNT})$ relies on the ability to fabricate electrodes contacting them. The major progress in the field has been largely attributed to the success in obtaining highly transparent contacts, with a small contact resistance compared to the quantum of resistance $\left(h / e^{2}=13 \mathrm{k} \Omega\right) .{ }^{1}$ The opposite case, a tunneling contact of low transparency has been given little systematic attention. Tunneling contacts, however, are useful for investigating the density of states via transport measurements and in various device applications.

In many earlier works the high measured resistance was attributed to unintentional tunneling contacts between a metal electrode and the CNT, the "bad" contact being due to poor wetting of the CNT by the metal. ${ }^{2}$ In semiconducting single walled nanotubes (SWNT) a Schottky barrier is believed to be formed between the tube and the metal electrode that acts as a tunneling barrier, ${ }^{3}$ but to date very few attempts have been made to construct tunneling contacts to CNTs, with thin insulating barriers. A measurement with a Scanning Tunneling Microscope (STM) is of course a measurement of tunneling conduction over a vacuum barrier, and besides the imaging of the nanotube wall, the band structure of SWNTs has been explored with this technique. ${ }^{4}$ The STM, however, is a very special set-up, and in two or more terminal devices precisely and reproducibly engineered tunneling contacts have to our knowledge not been achieved.

The standard tunneling device, a $M 1 / I / M 2$ junction, consists of two metal thin films, $M 1$ and $M 2$, which are separated by a thin insulating layer $(I)$ at most a couple of nanometers thick, as is schematically shown in Fig. 1(a). Experimental realization of a comparable scheme in which the other metal is replaced by a CNT is more cumbersome to achieve. Usually, the fabrication procedures for working tunnel junctions rely on deposition steps of the different thin films that are made in a high vacuum environment, uninterrupted by exposure to atmospheric conditions. On the other hand, the deposition process of CNTs is at some point incompatible with high vacuum environments.

There is also the question of how the geometry of a tunneling contact to the rounded shape of the CNT is arranged. To deposit by conventional methods a smooth and nanometerthin insulating barrier layer all around a section of the nanotube is certainly difficult. One possibility is growth by atomic layer deposition. ${ }^{5}$ A different but very simple approach is pictured in Fig. 1(b). A CNT is placed on top of a non-noble metal $M$ covered by its native oxide $\left(M_{\mathrm{OX}}\right)$, which will act as the insulating layer $I$. Then the tunneling contact is along the line where the CNT touches the $M / M_{\mathrm{OX}}$ surface.

We followed the idea of Fig. 1(b) by fabricating and measuring in this work such $M / M_{\mathrm{OX}} / \mathrm{CNT}$ junctions. The CNTs were multiwalled nanotubes not expected to have semiconducting gaps. The CNT was contacted from the other end with a Palladium (Pd) electrode, which is known to form good, low resistive, contacts to CNTs. ${ }^{2}$ For additional insight, we also made $M / M_{\mathrm{OX}} / \mathrm{Pd}$, that is, the $M 1 / I / M 2$-type of junctions of Fig. 1(a). As we will show, the $M / M_{\mathrm{OX}} / \mathrm{CNT}$ tunneling configuration allows for an easy exposure of the CNT, including the junction area, to different gaseous atmospheres, which in the case of oxygen proves to have a strong effect on the current-voltage (IV) characteristics (current I not to be confused with the same abbreviation for an insulator layer). Moreover, with the aim of understanding the role of the work function $(W)$ variations on the conductance of tunneling contacts to CNTs, we performed with the Kelvin probe force microscope (KPFM) measurements ${ }^{36}$ in different gaseous environments. The KPFM measures the difference between the local $W$ of the sample and the $W$ of the conductive tip of the KPFM. Prior to our work, the KPFM was successfully used to study charge-transfer processes at the Au-CNT junctions. ${ }^{37}$ 


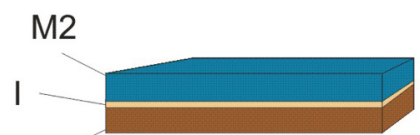

M1

(a)

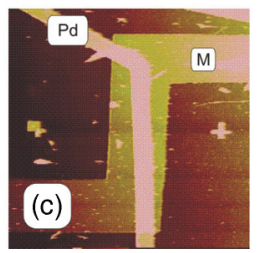

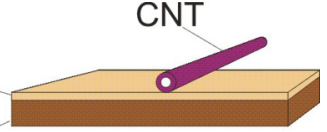

(b)

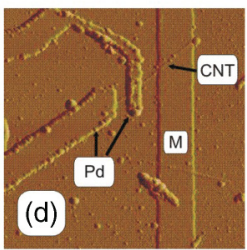

FIG. 1. (Color online) Schematic drawing of the two types of tunneling junctions used in this work. (a) A metal/insulator/metaljunction $(M / I / M)$, where the two metals $(M 1$ and $M 2)$ are different. (b) A metal/insulator/CNT junction where the insulator is the native oxide $\left(M_{\mathrm{ox}}\right)$ of the metal. (c) AFM image (topography signal) of a fabricated $M / M_{\mathrm{OX}} / \mathrm{Pd}$ junction. This junction is relatively large in size. (d) AFM image (error signal) of a $M / M_{\mathrm{OX}} / \mathrm{CNT}$ junction. The metal line $(M)$ is $1-\mu \mathrm{m}$ wide.

Finally, we have also fabricated and measured CNT field effect transistors (CNT-FET) of a type that contain the different junctions mentioned previously.

\section{EXPERIMENTAL}

The metals $M$ used in this work were non-noble ones and are therefore covered by a native oxide that forms immediately upon exposure to air after metallization. The metals that we have used are listed in Table I below, along with the energy gaps $\left(E_{\mathrm{g}}\right)$ of their (presumed) native oxides.

Two types of structure were fabricated, $M / M_{\mathrm{OX}} / \mathrm{Pd}$ and $M / M_{\mathrm{OX}} / \mathrm{CNT}$ junctions, both with the different metals listed in Table I. In both cases the fabrication begins with deposition of the metal $(M)$ lines, $10-\mathrm{nm}$ thick and $1-\mu \mathrm{m}$ wide, by standard $e$-beam lithography on a piece of $\mathrm{Si} / \mathrm{SiO}_{2}$ wafer. This $M$-electrode is then covered by its native oxide, $M_{\mathrm{OX}}$. For the $M / M_{\mathrm{OX}} / \mathrm{Pd}$ structures, the Pd electrodes (30-nm-thick) were deposited via alignment to partially overlap the $M$ lines in a second $e$-beam lithographic step. For the $M / M_{\mathrm{OX}} / \mathrm{CNT}$ junctions, multiwalled $\mathrm{CNTs}^{7}$ were dispersed by spin coating from dichloroethane solution on the $M$ structures. Such cases where one part of the CNT was on top of the $M$ line and the other outside it were searched with AFM and selected for further processing, in which one or two 30-nm-thick Pd electrodes were fabricated with a second step of electron beam lithography on the "free" end of the CNT. AFM images of $M / M_{\mathrm{OX}} / \mathrm{Pd}$ and $M / M_{\mathrm{OX}} / \mathrm{CNT}$ junctions are shown

TABLE I. Metals used in this work for the base electrode depicted in Fig. 1. Their presumed native oxides $\left(M_{\mathrm{OX}}\right)$ are listed, and the values for the energygap of the oxide $E_{\mathrm{g}}$ are indicated (Ref. 6).

\begin{tabular}{lcc}
\hline \hline Metal $(M)$ & Oxide $\left(M_{\mathrm{OX}}\right)$ & $E_{\mathrm{g}}$ \\
\hline Aluminum (Al) & $\mathrm{Al}_{2} \mathrm{O}_{3}$ & $8.3 \mathrm{eV}$ \\
Titanium (Ti) & $\mathrm{TiO}_{2}$ & $3.0 \mathrm{eV}$ \\
Niobium $(\mathrm{Nb})$ & $\mathrm{Nb}_{2} \mathrm{O}_{5}$ & $3.4 \mathrm{eV}$ \\
\hline \hline
\end{tabular}

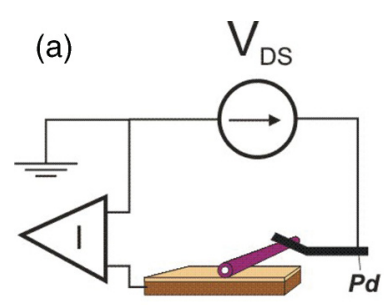

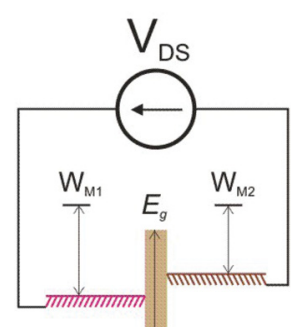

(b)

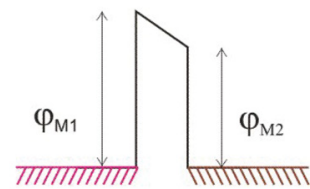

(c)
FIG. 2. (Color online) (a) The measurement scheme of the $M / M_{\mathrm{OX}} / \mathrm{CNT}$ junction. $M, M_{\mathrm{OX}}$, and CNT are as shown in Fig. 1(b). The voltage $\left(V_{\mathrm{DS}}\right)$ is applied with a voltage source to the palladium (Pd) electrode that contacts the CNT and the current (I) through the circuit measured with a current-voltage amplifier. In the $M / M_{\mathrm{OX}} / \mathrm{Pd}$ junction the Pd electrode connects directly to the $M / M_{\mathrm{OX}}$ electrode. (b) Generalized energy level scheme for the junctions discussed here, before lineup of Fermi levels. The work functions of the two electrodes $M 1$ and $M 2, W_{\mathrm{M} 1}$, and $W_{\mathrm{M} 2}$, respectively, are indicated, along with the energy gap $E_{\mathrm{g}}$ of the insulator $\left(M_{\mathrm{OX}}\right)$ between them. (c) At equilibrium, an asymmetric potential barrier separates the two electrodes of (b). The barrier heights $(\varphi)$ on both sides are indicated.

in Fig. 1(c) and 1(d), respectively. IV characteristics of the junctions were measured by biasing with a voltage source $\left(V_{D S}\right)$ and the current $(I)$ measured with a current amplifier. The measurement circuit for the $M / M_{\mathrm{OX}} / \mathrm{CNT}$ junction, including the contacts to the $\mathrm{Pd}$ and $M$ electrodes, is shown schematically in Fig. 2(a). The measurements of the IV characteristics of the junctions were performed in air at ambient pressure, in vacuum, and in pure $\mathrm{O}_{2}, \mathrm{~N}_{2}, \mathrm{H}_{2}$, and $\mathrm{CO}$.

KPFM measurements were carried out at room temperature by a Dimension 3100 Scanning Probe Microscope (SPM) (Bruker) equipped with a NanoScope 3D controller and operated in the "lift mode" using PPP-EFM probes from Nanosensors. In the lift mode measurements are taken in two passes across each scan line. In the first pass, topographical data is taken in normal tapping mode. Then the tip is raised to the lift scan height (typically $20-100 \mathrm{~nm}$ ), and a second scan is performed while maintaining a constant separation between the tip and local surface topography. The local work function distribution based on the Kelvin probe method ${ }^{36}$ during the second pass is recorded. In the Kelvin probe method the electrostatic tip-sample interaction, which stems from a difference in work function between tip and sample, is minimized by balancing voltage applied to the tip. In these measurements, all electrodes of the junction were connected to the ground. KPFM measurements were also performed to measure the potential distribution when an external voltage was applied across the junction. The SPM was enclosed in a glove box to control the gas environment: dry air (humidity < $10 \%$ ) or nitrogen gas atmosphere. 


\section{RESULTS}

\section{A. $M / M_{\mathrm{OX}} / \mathrm{Pd}$ junctions}

The $M / M_{\mathrm{OX}} / \mathrm{Pd}$ junctions were made with different areas within the range $0.1-35 \mu \mathrm{m}^{2}$. Of the several junctions that we fabricated a small minority exhibited Ohmic behavior, with a small resistance, and with very modest temperature dependence. We discarded these samples as cases with pinholes between the $M$ and the $\mathrm{Pd}$. In $M / M_{\mathrm{OX}} / \mathrm{Pd}$ junctions with aluminum as the metal $M$ we did not observe any current at moderate voltages. Only at voltages above $\sim 2 \mathrm{~V}$ did breakthrough events occur, whereby the current suddenly jumped by orders of magnitude and apparently irreversible damage to the oxide layer occurred as the junctions became electrically conducting. When the metal electrode was made from $\mathrm{Ti}$ or $\mathrm{Nb}$, the circuit was regularly conducting at moderate bias levels, and the IV curves were reversible. In the following we will consider exclusively $\mathrm{Ti}$ and $\mathrm{Nb}$ junctions.

Figure 3(a) shows room temperature IV curves for $\mathrm{Ti} / \mathrm{Ti}_{\mathrm{OX}} / \mathrm{Pd}$ junctions of different sizes (areas) and one $\mathrm{Nb} / \mathrm{Nb}_{\mathrm{OX}} / \mathrm{Pd}$ junction. Though there are not many cases to judge from, the latter seem to be more resistive (lower current per junction area). The IV curves are strongly nonlinear and also quite asymmetric. For the $\mathrm{Ti} / \mathrm{Ti}_{\mathrm{OX}} / \mathrm{Pd}$ samples we found that if we normalize the current with the junction area then the current density is still larger in larger area junctions. This has the natural explanation in that the oxides are not truly uniform but have randomly dispersed thinner sections through which much larger tunneling currents flow than elsewhere. In larger junctions these strong connections are then encountered more likely. The $\mathrm{Nb}$ junctions show no consistent behavior in this respect, but we had fewer samples of them. The nonuniformity of practical $M / I / M$ junctions has been discussed in Ref. 9.

The temperature dependence for a $\mathrm{Ti} / \mathrm{Ti}_{\mathrm{OX}} / \mathrm{Pd}$ junction is shown in Fig. 3(b). In all samples the dependence was monotonic with increasing resistance with decreasing temperature. For the zero-bias resistance the temperature dependence is very strong. The IV curves of the $M / M_{\mathrm{OX}} / \mathrm{Pd}$ junctions are almost independent of the gas environment.
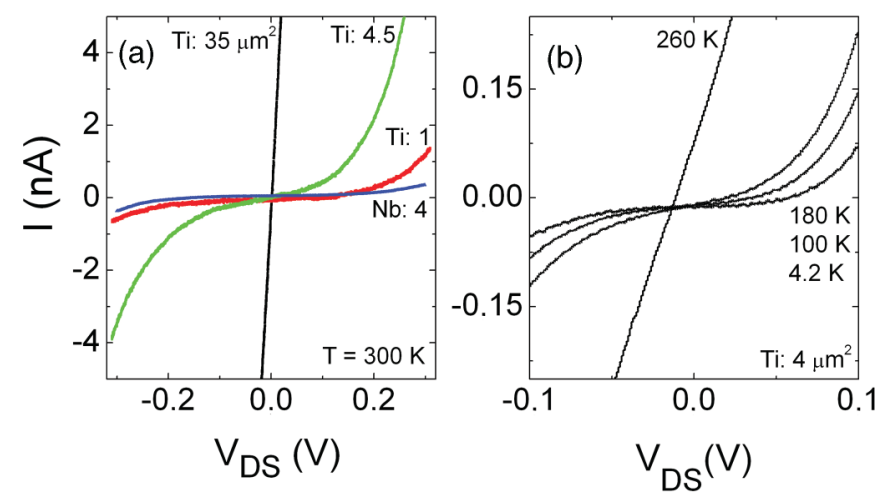

FIG. 3. (Color online) (a) IV curves at room temperature of $\mathrm{Ti} / \mathrm{Ti}_{\mathrm{OX}} / \mathrm{Pd}$ junctions of different areas and one $\mathrm{Nb} / \mathrm{Nb}_{\mathrm{OX}} / \mathrm{Pd}$ junction. The area is given for each sample in $\mu \mathrm{m}: \mathrm{s}$. (b) Temperature dependence of a $\mathrm{Ti} / \mathrm{Ti}_{\mathrm{OX}} / \mathrm{Pd}$ junction of area $4 \mu \mathrm{m}^{2}$ at small voltages.

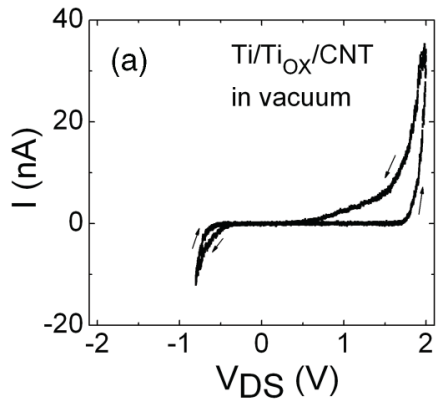

(c)

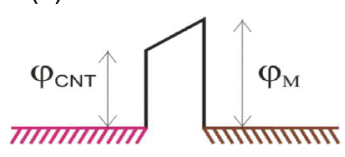

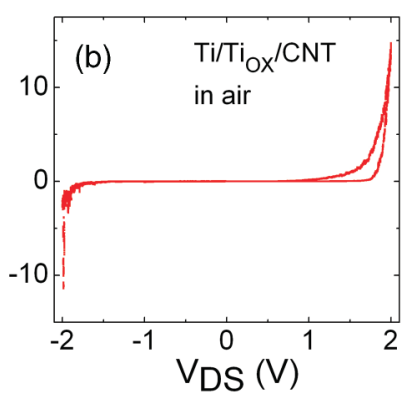

(d)

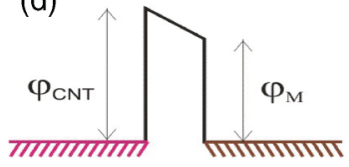

FIG. 4. (Color online) The room temperature IV characteristics of a representative $\mathrm{Ti} / \mathrm{Ti}_{\mathrm{OX}} / \mathrm{CNT}$ junction in (a) vacuum and in (b) air. (a) The arrows indicate the direction of the voltage sweep. (c), (d) Suggested energy barrier height scheme corresponding to the cases above (where $M=\mathrm{Ti}$ ) in (c) vacuum and in (d) $\mathrm{O}_{2}$.

\section{B. $M / M_{\mathrm{OX}} / \mathrm{CNT}$ junctions: Transport measurements}

The measurement circuit for $M / M_{\mathrm{OX}} / \mathrm{CNT}$ junctions is shown in Fig. 2(a). Considering the circuit, we have the following resistances to consider as parts of the overall measured resistance: the $\mathrm{Pd}$ and $\mathrm{M}$ electrodes, the $\mathrm{Pd} / \mathrm{CNT}$ contact, the CNT, and the $M / M_{\mathrm{OX}} / \mathrm{CNT}$ junction. The three first ones fall within the range of $0.1-100 \mathrm{k} \Omega$ (on CNT contact resistances, Refs. 2 and 17-19; on multiwalled CNT intrinsic resistivity, Refs. 19 and 20-23). Since the measured zero bias resistances vary in the range from $M \Omega$ :s to $G \Omega$ :s, we can conclude that the other resistances are negligible compared to the fourth and last one, and therefore the resistance of the circuit is essentially that of the $M / M_{\mathrm{OX}} / C N T$ junction. As is shown in Fig. 1(d), we have in some of the samples added an extra electrode to measure the resistance of the CNTs with two Pd electrodes, where we find an ohmically behaving resistance of a few tens of $\mathrm{k} \Omega$ :s.

In Fig. 4 the IV characteristics of a Ti/Ti $\mathrm{OX}_{\mathrm{O}} / \mathrm{CNT}$ junction in (a) vacuum and (b) in air at ambient pressure (b) are shown. Examining first the IV curve in vacuum, we find it to be repeatable but also observe a significant hysteretic behavior; at positive voltages the return current is higher, while at negative voltages it is lower. The hysteresis was similar in all samples. The IV curves are asymmetrical; the minimum conductance, though unmeasurable due to the high resistance, is clearly on the positive side. Among the $\sim 20 \mathrm{M} / \mathrm{M}_{\mathrm{OX}} / \mathrm{CNT}$ junctions that we fabricated $(M=\mathrm{Ti}, \mathrm{Nb})$, we did not encounter a single case of a short cut between the CNT and the metal electrode, as we did among the $M / M_{\mathrm{OX}} / \mathrm{Pd}$ junctions. While the $M / M_{\mathrm{OX}} / \mathrm{Pd}$ junctions were much more conductive and nonhysteretic, in $M / M_{\mathrm{OX}} / \mathrm{CNT}$ junctions a higher voltage is needed for an observable current. The magnitudes of the applied voltages are also of significance. Consider the positive side of the IV curve, which reaches up to $2 \mathrm{~V}$. The electric field strength is then of the order of $10^{7} \mathrm{~V} / \mathrm{cm}$, which certainly approaches the breakdown strength of the oxide. ${ }^{24}$ 


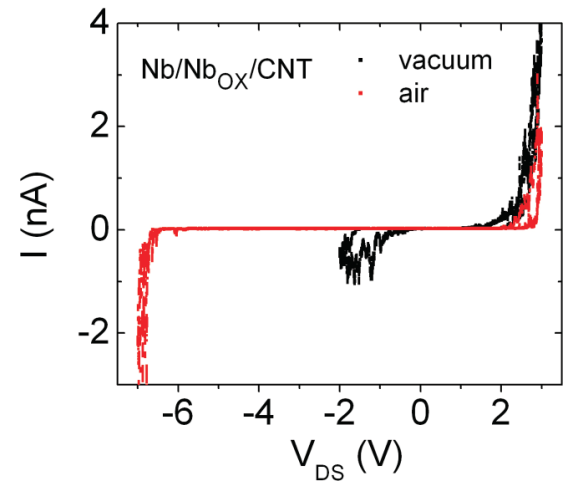

FIG. 5. (Color online) IV curve of a $\mathrm{Nb} / \mathrm{Nb}_{\mathrm{OX}} / \mathrm{CNT}$ junction in vacuum and in air of $1 \mathrm{~atm}$ pressure.

Air causes a large increase of the gap in the IV curve when compared to that of vacuum. The gap widens to the negative side of the IV curve and barely at all on the positive side. Now the asymmetry has changed polarity; the minimum conductance is on the negative side. This shift is due to the oxygen, since the shift is the same with pure $\mathrm{O}_{2}$, while pure $\mathrm{N}_{2}$, $\mathrm{H}_{2}$, and $\mathrm{CO}$ have no effect on the IV curve compared to that in a vacuum environment. The effect is reversible, repeated cycling between a vacuum and oxygen atmosphere do not change the behavior, with the exception that rapid changes may produce different outcomes. On the other hand the $\mathrm{Ti} / \mathrm{Ti}_{\mathrm{OX}} / \mathrm{Pd}$ junctions are, according to our measurements, almost independent of the gas environment, as was previously mentioned.

$\mathrm{Nb} / \mathrm{Nb}_{\mathrm{OX}} / \mathrm{CNT}$ junctions were also measured, with one representative example shown in Fig. 5. The same behavior can be seen as in the previous Ti-based system: The IV curve in vacuum is slightly asymmetrical with higher currents on the negative side. The effect of air is to significantly widen the gap to the negative side of the voltage scale. The size of the gap in oxygen in particular varies, but both in vacuum and in oxygen it is larger than in $\mathrm{Ti} / \mathrm{Ti}_{\mathrm{OX}} / \mathrm{CNT}$ junctions.

The temperature dependence of Ti/TiOX/CNT devices is demonstrated in Fig. 6, in vacuum and in 0.7 atm pressure of $\mathrm{O}_{2}$, for the negative side of the IV curves, where the effect of oxygen is much stronger. The difference between the two cases is striking: in vacuum, the currents consistently decrease
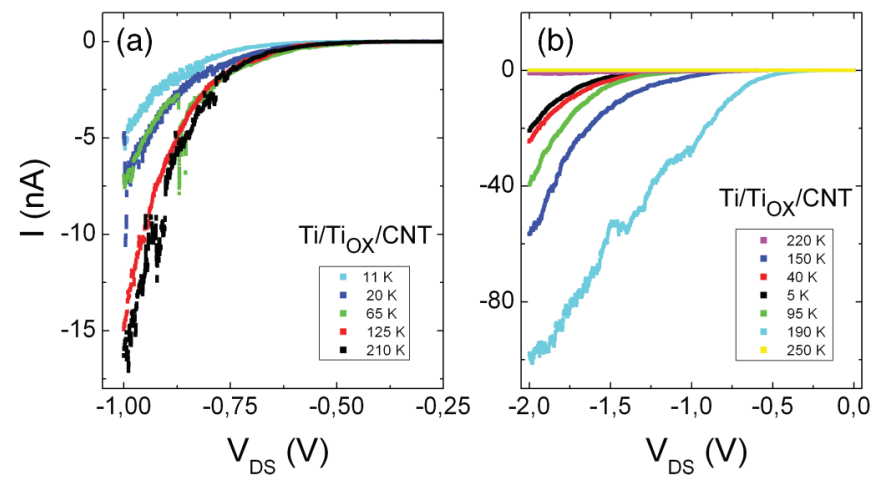

FIG. 6. (Color online) Temperature dependence of the IV characteristics of $\mathrm{Ti} / \mathrm{Ti}_{\mathrm{OX}} / \mathrm{CNT}$ junctions in (a) vacuum and (b) in $\mathrm{O}_{2}$ pressure $(0.7 \mathrm{~atm}$ at $300 \mathrm{~K})$. The sequence of the data at different temperatures is the one in which the measurements have been done. upon decreasing temperature; while in oxygen, the currents at first strongly increase with decreasing temperature, down to a temperature of $130 \mathrm{~K}$, and then begin to decrease as the sample cools further. Unfortunately, we do not have data of the temperature dependence in vacuum and oxygen performed on the same CNT device, but the trend is nevertheless well established from multiple measurements on several devices. As Fig. 6(b) shows, the behavior is fully reversible upon warming from cryogenic temperatures up to room temperature.

\section{C. $M / M_{\mathrm{OX}} / \mathrm{CNT}$ junctions: Kelvin probe microscopy measurements}

We have performed KPFM measurements on $\mathrm{Nb} / \mathrm{Nb}_{\mathrm{OX}} / \mathrm{CNT}$ devices, which are presented next. AFM topography images of the device are shown in Fig. 7(a) and (e). The $\mathrm{Nb} / \mathrm{Nb}_{\text {OX }}$ electrode $(1-\mu \mathrm{m}$ wide) is shown on the top-left corner of the image Fig. 7(e) and more closely in Fig. 7(a). The CNT is contacted from the other end with two Pd electrodes, which are displayed in the middle part/lower right of image Fig. 7(e).

The KPFM images of the device, taken at different conditions, are shown in Fig. 7(b) and (c) and Fig. 7(f) and (g). The KPFM image presented in Fig. 7(b) is acquired in a dry air atmosphere at zero voltage applied between the electrodes, whereas the image in Fig. 7(c) is acquired in a nitrogen atmosphere. These images map the work function distribution in the vicinity of the $\mathrm{Nb} / \mathrm{Nb}_{\mathrm{OX}} / \mathrm{CNT}$ junction. To verify whether the environment modifies the difference of
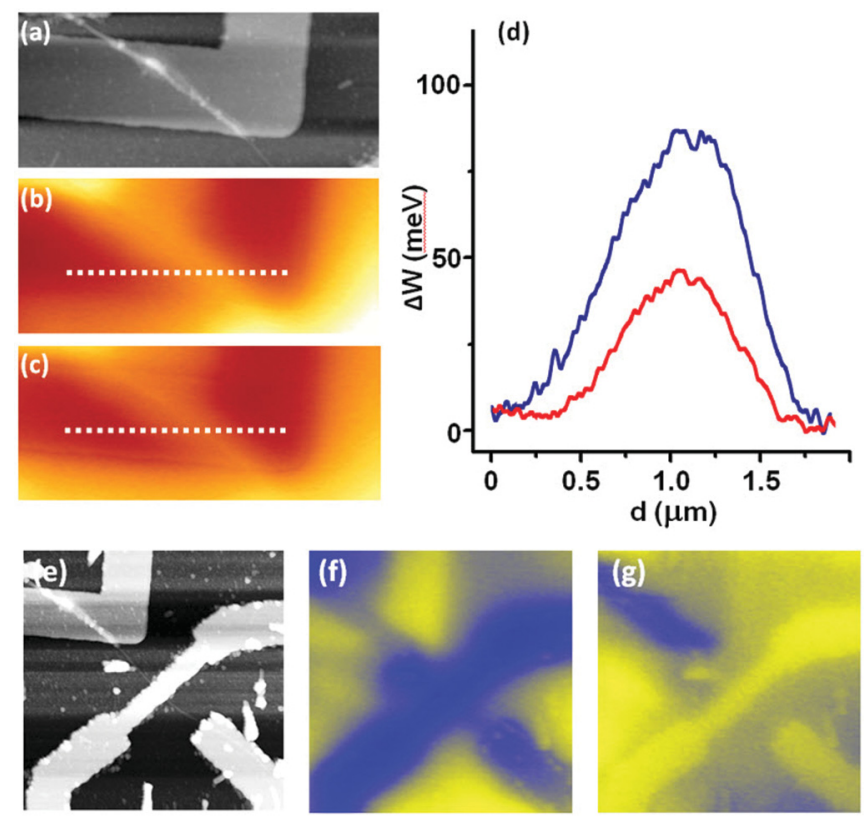

FIG. 7. (Color online) The examples of the results of KPFM measurements performed on the $\mathrm{Nb} / \mathrm{Nb}_{\mathrm{OX}} / \mathrm{CNT}$ device. (a) $4.5 \times$ $2 \mu \mathrm{m}^{2}$ AFM topography image of the CNT-Nb/Nb $/ \mathrm{Nx}_{\text {ox }}$ contact area of a device. Correspondent KPFM images acquired at $\mathrm{Vds}=0$ in (b) dry air and in (c) nitrogen gas atmosphere. Work function difference $\Delta W$ profiles measured along the dashed lines in (b), (c) in air and in (d) nitrogen. $5.5 \times 5.5 \mu \mathrm{m}^{2} \mathrm{AFM}$ image of the device (e) with corresponding KPFM images: acquired at applied voltage $\mathrm{Vds}=$ $-1 \mathrm{~V}$ (f) and acquired within $\sim 10 \mathrm{~min}$ after reducing Vds to zero (f). 
$W$ between the metal contact and the CNT, we compare in Fig. 7(d) the local KPFM potential profiles across the CNT obtained in dry air and in a nitrogen gas atmosphere. The measured drop of potential between the CNT and the $\mathrm{Nb}$ electrode is $\approx 80 \mathrm{mV}$ in a dry air atmosphere while it is almost twice less in a nitrogen atmosphere.

The KPFM images shown in Fig. 7(f) and (g) map the potential distribution in the CNT device in the current carrying state in a nitrogen gas atmosphere with applied voltage Vds $=-1 \mathrm{~V}$ and after reducing $\mathrm{Vds}$ to zero, respectively. The difference of potentials between the $\mathrm{Nb}$ and $\mathrm{Pd}$ electrodes at $\mathrm{Vds}=-1 \mathrm{~V}$ is clearly visible in Fig. 7(f). The CNT is almost equipotential with the Pd electrodes, whereas the voltage drops on the $\mathrm{Nb} / \mathrm{Nb}_{\mathrm{OX}} / \mathrm{CNT}$ junction confirm that practically all the resistance of the device is concentrated on the junction. The KPFM image presented in Fig. $7(\mathrm{~g})$ is acquired at $\mathrm{Vds}=0$. The difference of potentials between $\mathrm{Nb}$ and $\mathrm{Pd}$ electrodes is $\approx 0.4 / \mathrm{V}$. On the other hand, the potential map of the device [Fig. $7(\mathrm{~g})]$ reveals a considerable depression of the potential $\Delta U_{\mathrm{j}} \sim 0.7 \mathrm{~V}$ nearby the $\mathrm{Nb} / \mathrm{Nb}_{\mathrm{OX}} / \mathrm{CNT}$ junction. Such a depression can be considered as a "memory effect" because it is not observable without any preliminary applied voltage to the electrodes.

\section{DISCUSSION}

The junctions investigated in this work are of type $M 1 / I / M 2$ (electrodes of different material), which means that the observed asymmetry in the voltage-dependent conductance is expected. Such a (biased) junction is shown in the scheme of Fig. 2(b), where a metal oxide $\left(M_{\mathrm{OX}}\right)$ acts as the insulator $I$. The work functions $W_{\mathrm{M} 1}$ and $W_{\mathrm{M} 2}$, of $M 1$ and $M 2$, respectively, and the energy gap $E_{\mathrm{g}}$ of $M_{\mathrm{OX}}$ are indicated. The picture refers to the situation before alignment of the Fermi levels upon contact. The resulting energy level diagram, after equalization of Fermi levels, is shown in Fig. 2(c). The different barrier heights, $\varphi_{\mathrm{M} 1}$ and $\varphi_{\mathrm{M} 2}$, on the two sides of the oxide thus result in a trapezoidal, and asymmetric, energy barrier. If $\chi$ is the electron affinity of the barrier $M_{\mathrm{OX}}$, then a simple expression for the barrier height $\varphi$ on one side of the junction would be 8

$$
\phi=\mathrm{W}-\chi .
$$

That $M / M_{\mathrm{OX}} / \mathrm{Pd}$ and $M / M_{\mathrm{OX}} / \mathrm{CNT}$ junctions are asymmetrical junctions then follows from the differing energy barriers on the different sides of the insulator. The asymmetric IV characteristics follow from the asymmetry in the energy diagram, as, for example, has been discussed in the classical paper by Brinkman et $a l^{25}$ Generally, however, $\varphi$ in a $M 1 / I / M 2$ junction is not necessarily determined in such a straightforward way solely by the work functions and the energy gap. ${ }^{8,12}$

Neglecting the asymmetry in barrier shape, the simplest expression for direct tunneling in a $M / I / M$ junction gives the tunneling current as exponentially dependent on both thickness (d) and height of the energy barrier: ${ }^{10}$

$$
I \sim \exp [-2 \kappa d],
$$

where $\kappa=\sqrt{(} 2 m \phi) / \hbar$. Expression (2) is temperature independent, which is a characteristic of true direct tunneling conduction. In a more refined model a small temperaturedependent factor comes from the temperature dependence of the Fermi distribution. ${ }^{11}$ We do not have definite data on the thickness of our native oxides, but considering the values of $E_{\mathrm{g}}$ for the native oxides of these metals, listed in Table I, and assuming that the oxide thicknesses are the same, we could conclude that the higher energy barrier in $\mathrm{Al} / \mathrm{Al}_{\mathrm{OX}} / \mathrm{Pd}$ junctions is the likely cause for the difference in behavior, with respect to the $\mathrm{Ti}-$ and $\mathrm{Nb}$-based junctions. Also, the higher conductance of $\mathrm{Ti} / \mathrm{Ti}_{\mathrm{OX}} / \mathrm{Pd}$ junctions compared to the $\mathrm{Nb} / \mathrm{Nb}_{\mathrm{OX}} / \mathrm{Pd}$ junctions corresponds to the lower value of the $E_{\mathrm{g}}$ of $\mathrm{TiO}_{2}$ with respect to that of $\mathrm{Nb}_{2} \mathrm{O}_{5}$. However, since the dependence on oxide thickness is very strong as well, we can not make definite conclusions on this issue.

The zero-bias resistances of the $M / M_{\mathrm{OX}} / \mathrm{CNT}$ junctions were beyond the measurement range, while those of the $M / M_{\mathrm{OX}} / \mathrm{Pd}$ junctions are strongly temperature dependent, as was shown in Fig. 3(b). Though we have not measured accurately the temperature dependence, we can observe that it is exponentially strong, leading to a vanishing zero-bias conductance. We may conclude that the conduction is not pure direct tunneling but due to thermally assisted tunneling processes within the oxide or at the interfaces. $M / I / M$-type of junctions where the insulator is a native oxide and can be demonstrated to work on direct tunneling, are made via carefully controlled oxidation inside vacuum evaporation equipment. ${ }^{12}$ These junctions have an oxide thickness $<2 \mathrm{~nm}$, while our systems, where the oxidation is via exposure to ambient atmosphere, will be thicker than this. The actual conduction mechanism then is very difficult to pinpoint precisely. One possibility is thermionic emission of charge carriers over the barrier. ${ }^{13,14}$ Another is sequential hopping through localized states through defect sites within the oxide. This is particularly plausible considering that our native oxide is certainly defective. In the latter case, if the average hopping distance were much less than the oxide thickness, we would obtain the well known Variable Range Hopping (VRH) model. ${ }^{15}$ However, we consider it unlikely with only a native oxide thickness of a couple of nanometers. The intermediate case, with the barrier thickness too large for direct tunneling but smaller than the VRH hopping distance, was examined thoroughly in the work by Xu et al. ${ }^{16}$ Our system is likely to be of this type; the dominating current stems from charge carriers undergoing a very few thermally assisted tunneling steps. A complete theoretical description would need to take into account the temperature dependence of the conduction and also the asymmetry of the junction IV characteristics, which is usually described in connection with direct tunneling conduction. We are not aware of such work.

We will now concentrate on the main topic of this work, the $M / M_{\mathrm{OX}} / \mathrm{CNT}$ junctions. A detailed, quantitative modeling of the IV characteristics is difficult even for pure direct tunneling junctions and perhaps counterproductive in this case when the precise mechanism can not be pinpointed, and because we are approaching breakdown levels at the highest applied voltages, as was noted previously. We may, however, qualitatively describe the asymmetry in the data on $M / M_{\mathrm{OX}} / \mathrm{CNT}$ junctions. Reference 25 gives an expression for the voltage $V_{\min }$ at which the conductance minimum occurs. Applied to the circuit 
configuration in Fig. 2(b), this expression would be

$$
V_{\min } \sim \frac{\varphi_{M}-\varphi_{C N T}}{\sqrt{\bar{\varphi}}},
$$

where $\bar{\varphi}$ is the average barrier height. Upon inspection of Fig. 4(a), we can note that the conduction minimum, though not accurately measurable due to the high resistance, would be at a positive voltage. Equation (3) would then yield the relation $\varphi_{\mathrm{M}}>\varphi_{\mathrm{CNT}}$ and the corresponding energy scheme, as depicted in Fig. 4(c).

Although, as was stated previously, values for work functions $(W)$, do not translate straightforwardly into barrier values $(\varphi)$, we may attempt to equate the expression $\varphi_{\mathrm{M}}-\varphi_{\mathrm{CNT}}$ in Eq. (3) with the corresponding differences in $W$ and so obtain some estimates for the asymmetry of the IV characteristics in terms of the sign of $V_{\min }$. Work function values are available for the different metals ${ }^{26}$ and some rather recent measurements for CNTs. ${ }^{27}$ These are for Pd: $5.22-5.6 \mathrm{eV}$; Ti: $4.33 \mathrm{eV}$; $\mathrm{Nb}$ : $3.95-4.87 \mathrm{eV}$; and for a multiwalled CNT: $4.95 \mathrm{eV}$. The measured potential difference in the KPFM measurements is in a reasonable agreement with the $W$ values for $\mathrm{Nb}$ and $\mathrm{Pd}$, while for the transport measurements, $V_{\min } \sim \varphi_{\mathrm{M}}-\varphi_{\mathrm{Pd}} \sim$ $W_{\mathrm{M}}-W_{\mathrm{Pd}}$, which is negative for both $\mathrm{Ti}$ and $\mathrm{Nb}$. As we can see from Fig. 3, the $M / M_{\mathrm{OX}} / \mathrm{Pd}$ junctions indeed have the conductance minimum on the negative side of the IV curve. For the $M / M_{\mathrm{OX}} / \mathrm{CNT}$ junctions, on the other hand, $V_{\min }$ in vacuum is clearly negative, while the previous formula would predict a slightly negative value still. This would then indicate that the barrier height at the interface between the CNT and the $M_{\mathrm{OX}}$ is affected also by other factors besides the work function.

Upon exposure to air the IV curves change quite drastically; the asymmetry switches polarity as the gap region of the IV curve now extends much further on the negative side [Fig. 4(b)]. On the positive side there is only a barely discernible extension of the gap. The voltages on the negative side are now even larger, especially for $\mathrm{Nb}$, and, indeed, the IV characteristics on the negative side resemble even more now a breakdown current, being still reproducible, however. The suggested energy scheme in this new situation is depicted in Fig. 4(d), in which now $\varphi_{\mathrm{M}}<\varphi_{\mathrm{CNT}}$. These results are in agreement with the results of the KPFM measurements of the $W$ distribution in the vicinity of the $\mathrm{Nb} / \mathrm{Nb}_{\mathrm{OX}} / \mathrm{CNT}$ junction. Indeed, the measured $W$ difference between the CNT and the $\mathrm{Nb}$ electrode in a dry air atmosphere is almost twice larger than in a nitrogen atmosphere [Fig. 7(d)]. Although $W$ does not translate straightforwardly into $\varphi$, reduction of $\Delta W$ in a nitrogen gas atmosphere clearly correlates with the correspondent decrease of the barrier heights difference $\varphi_{\mathrm{CNT}}$ $-\varphi_{\mathrm{M}}$, [Fig. 4(c), (d)].

It should be noted that the KPFM measurements also shed light on the hysteretic behavior, which was observed in the IV curves (Figs. 4 and 5). The KPFM measurements performed at different voltages applied across the junction and presented in Fig. 7(f) and (g) revealed a "memory effect," which can be attributed to the charge injection from the CNT to the oxide layer. The charged $\mathrm{Nb}_{\mathrm{OX}}$ region nearby the junction serves as a "gate" which modifies the return current in the IV characteristics of the $M / M_{\mathrm{OX}} / \mathrm{CNT}$ junctions and thereby causes the hysteresis. The charge injection was observed as the decay of $\Delta U_{\mathrm{j}}$ with a time constant of $\sim 40 \mathrm{~min}$. The "memory effect" is also observed at positive values of $V_{\mathrm{DS}}$ but is much smaller.

The temperature dependence of the conduction in $M / M_{\mathrm{OX}} / \mathrm{CNT}$ junctions was shown in Fig. 6. To recapitulate, the currents were monotonously decreasing in vacuum while in oxygen they at first increased and then, around $T=$ $100 \mathrm{~K}$, began to decrease again. This development is roughly coincidental with the decrease of oxygen pressure upon cooling. Oxygen has a boiling point of $90 \mathrm{~K}$. At $100 \mathrm{~K}$ we have recorded a zero pressure, meaning that the sample effectively is in vacuum. Thus we apparently observe the vanishing of the doping effect of the oxygen, due to its liquefaction, and the IV characteristics of the device become similar to that in vacuum. We can thus conclude that the interaction of oxygen with the CNT at lower temperatures is a fully reversible gas phase reaction.

As we mentioned previously, our experiment indicates that the shift in the IV characteristics by air exposure is caused by oxygen, with other gases such as $\mathrm{N}_{2}, \mathrm{CO}$, and $\mathrm{H}_{2}$ having no effect compared to the vacuum condition. Oxygen doping of CNTs have been observed to significantly affect the thermopower of CNT films. ${ }^{28}$ In photoluminescence the effect of oxygen absorption was seen, but the precise origin is unclear. ${ }^{29}$ For individual SWNTs, the effect of oxygen effects have been studied in CNT-FETs. ${ }^{38}$

Oxygen is an electron acceptor. The interaction between $\mathrm{CNT}$ and $\mathrm{O}_{2}$ has been theoretically considered. ${ }^{30}$ An electron transfer from the CNT to $\mathrm{O}_{2}$ results in hole doping of the CNT and a lowering of the Fermi level. Thus the work function of the $\mathrm{CNT}\left(\varphi_{\mathrm{CNT}}\right)$ also increases, and if the increase is large enough we may have for the $M / M_{\mathrm{OX}} / \mathrm{CNT}$ junction the situation depicted in Fig. 4(d), where the asymmetry of the junction has changed polarity. Therefore we could understand the change in the IV curves upon exposure to oxygen gas as caused by hole doping of the CNT, which reverses the relative size of the tunneling barriers in the $M / M_{\mathrm{OX}} / \mathrm{CNT}$ junction as $\varphi_{\mathrm{CNT}}$ becomes larger than $\varphi_{\mathrm{M}}$. While some studies do not support a simple picture of oxygen gas doping of $\mathrm{CNTs},{ }^{39}$ it is unambigously known that defective sites in CNTs absorb more readily and react more in the way of chemisorption than physisorption, the former involving more readily charge transfer than the latter. The contact region between the CNT and the $M_{\mathrm{OX}}$ surface could conceivably be more prone to absorption of oxygen than the unperturbed parts of the CNT and thus dominate the response of the device to changes in the gaseous environment.

In the case of CNT-FETs with individual SWNTs, the interpretation of the available experimental data may be more complicated. ${ }^{3,31}$ It is argued that the oxygen affects the metal work function at the interface between the metal electrode and the CNT rather than via doping of the CNT. However, since in our case the $M / M_{\mathrm{OX}} / \mathrm{Pd}$ junctions showed no or very little effect, we prefer the view of doping via the CNT, although we can not at this stage rule out alternative scenarios.

\section{A. CNT-based FETs}

Finally, we will apply the information obtained previously on the $M / M_{\mathrm{OX}} / \mathrm{Pd}$ and $M / M_{\mathrm{OX}} / \mathrm{CNT}$ junctions to the related 

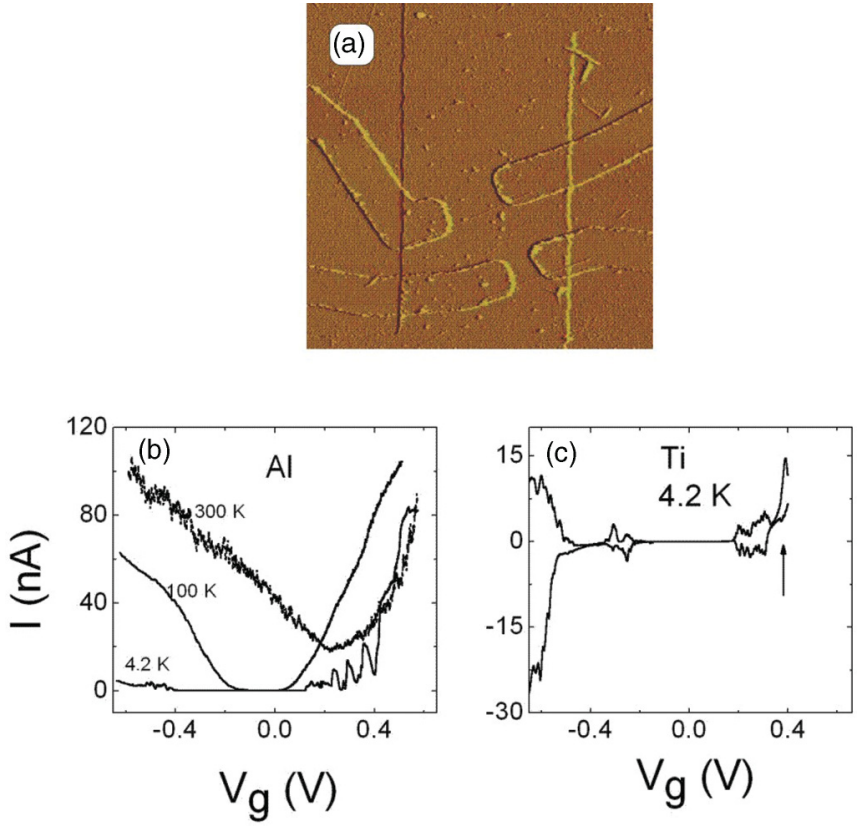

FIG. 8. (Color online) (a) AFM image of two CNT-FETs. Two CNTs on a $1-\mu$ m-wide $\mathrm{Al} / \mathrm{Al}_{2} \mathrm{O}_{3}$ gate electrode, which is vertically directed in the image. The CNTs are contacted by Pd electrodes. (b) Current (I) vs gate voltage $\left(V_{g}\right)$ at different temperatures for a CNT-FET with an $\mathrm{Al} / \mathrm{Al}_{2} \mathrm{O}_{3}$ gate. Bias voltage $V_{\mathrm{DS}}$ is $50 \mathrm{mV}$. (c) I vs $V_{g}$ at $V_{\mathrm{DS}}$ of $+10 \mathrm{mV}$ and $-10 \mathrm{mV}$ for a CNT-FET with a $\mathrm{Ti} / \mathrm{TiO}_{2}$ gate. Temperature is $4.2 \mathrm{~K}$. The arrow points the beginning of the gate leakage in the Ti-based structure.

topic of highly proximate backgates in CNT-FETs. Such are desired, which would have a high capacitive connection to the CNT and therefore be an efficient gate. One frequently used method is to deposit the CNT on a wide metal gate electrode, where the two are separated from each other with a thin insulator, which can be the native oxide ${ }^{23,32-34}$ or a separately deposited insulating molecular layer. ${ }^{31,35}$ In both cases one crucial question is, just as in modern silicon FET design, whether the insulating layer is able to prevent tunneling or breakdown current to the CNT as the gate voltage is applied. The aim is then, in a way, exactly opposite to the work described previously.

We have tested CNT-FETs with CNTs placed completely on wide $\mathrm{Al}$ and $\mathrm{Ti}$ electrodes, which act as gate electrodes with the native oxide as the gate insulator, as is shown in Fig. 8(a). The 200-nm-wide Pd drain and source electrodes extended over the gate electrodes to the CNTs. For the CNTFET to work properly, both the CNT and the Pd electrode must not leak through the gate insulator as gate or drain-source voltages are applied. In other words, we may take advantage of the knowledge in the previous experiments on $M / M_{\mathrm{OX}} / \mathrm{Pd}$ and $M / M_{\mathrm{OX}} / \mathrm{CNT}$ junctions to construct a working CNT-FET based on metal electrode insulated by its own native oxide.

The results are shown in Figs. 8(b) and 7(c) for CNT-FETs with $\mathrm{Al}$ and $\mathrm{Ti}$ gate electrodes, respectively. As is expected from the previous results, the CNT-FET with an Al gate worked well at all temperatures and did not leak to the gate up to 1-2 $\mathrm{V}$ of applied gate voltage. The CNT-FET with an Ti gate then could not be properly operated at room temperature, but at $4.2 \mathrm{~K}$ clear gate modulation could be observed at modest gate voltages. In right side of Fig. 8(c), an arrow points to the positive gate voltage where the $\mathrm{TiO}_{2}$ insulator begins to leak. On the other hand, at negative voltages of the same magnitude there are still no major signs of leak currents. We can thus conclude that the separately measured data from the $M / M_{\mathrm{OX}} / \mathrm{Pd}$ and $M / M_{\mathrm{OX}} / \mathrm{CNT}$ junctions are consistent with the CNT-FET data.

\section{SUMMARY}

We have measured the conductance of tunneling contacts to CNT, where the tunneling barrier is the native oxide of the metal electrode. The quality of these oxides is far from ideal as the tunneling conduction mechanism is somewhere between direct tunneling and hopping conduction. However, the intrinsic properties, presumably the work function, of the metal and the CNT are responsible for the asymmetry of the IV curve. This asymmetry switches polarity as oxygen gas is introduced to the junction. Our preferred conclusion is that the oxygen hole dopes the CNT and thus changes its work function, but we can not at this point rule out alternative scenarios such as changes via the metal work function. Our conclusion is in agreement with the results of the Kelvin probe microscopy measurements of the work function distribution in the vicinity of the junctions in a dry air and in a nitrogen gas atmosphere. This kind of junction seems promising as a way to probe the oxygen-doping process of CNTs in more detail or of some other dopant species than has been done to date. Future work, however, should find a fabrication method that produces junctions where the conduction is dominated by direct tunneling. Very recently, in connection with the rapid progress in graphene research, single atom thick layers of insulating 2D layered materials such as $\mathrm{BN}$ have been produced. ${ }^{40}$ These have been applied as tunneling barriers and may also be used for the purpose in this article.

\section{ACKNOWLEDGMENTS}

We thank Karsten Albe from T.U. Darmstadt for his advice. This research was supported by the Academy of Finland.
*Corresponding author: ahlskog@jyu.fi

${ }^{1}$ A. Jorio, M. S. Dresselhaus, and G. Dresselhaus, eds., Carbon Nanotubes, Vol. 111, Topics in Applied Physics (Springer-Verlag, Berlin, 2008).

${ }^{2}$ D. Mann, A. Javey, J. Kong, Q. Wang, and H. Dai, Nano Lett. 3, 1541 (2003).
${ }^{3}$ S. Heinze, J. Tersoff, R. Martel, V. Derycke, J. Appenzeller, and P. Avouris, Phys. Rev. Lett. 89, 106801 (2002).

${ }^{4}$ J. W. G. Wildöer, L. C. Venema, A. G. Rinzler, R. E. Smalley, and C. Dekker, Nature 391, 59 (1998).

${ }^{5}$ D. B. Farmer and R. G. Gordon, Nano Lett. 6, 699 (2006). 
${ }^{6}$ J. Portier, H. S. Hilal, I. Saadeddin, S. J. Hwang, M. A. Subramanian, and G. Campet, Prog. Solid State Chem. 32, 207 (2004).

${ }^{7}$ A. Koshio, M. Yudasaka, and S. Iijima, Chem. Phys. Lett. 356, 595 (2002).

${ }^{8}$ R. B. Floyd and D. G. Walmsley, J. Phys. C 11, 4601 (1978).

${ }^{9}$ C. W. Miller, Z.-P. Li, J. Åkerman, and I. K. Schuller, Appl. Phys. Lett. 90, 043513 (2007).

${ }^{10}$ C. Julian Chen, Introduction to Scanning Tunneling Microscopy, 2nd ed. (Oxford University Press, New York, 2008).

${ }^{11}$ E. L. Wolf, Principles of Electron Tunneling Spectroscopy (Oxford University Press, New York, 1985).

${ }^{12}$ K. Gloos, P. J. Koppinen, and J. P. Pekola, J. Phys.: Condens. Matter 15, 1733 (2003).

${ }^{13}$ H. Im, Yu. A. Pashkin, T. Yamamoto, O. Astafiev, Y. Nakamura, and J. S. Tsai, Appl. Phys. Lett. 88, 112113 (2006).

${ }^{14}$ S. M. Sze, Physics of Semiconductor Devices (Wiley, New York, 1981).

${ }^{15}$ N. F. Mott and E. A. Davis, Electronic Processes in Noncrystalline Materials (Oxford University Press, Oxford, 1979).

${ }^{16}$ Y. Xu, D. Ephron, and M. R. Beasley, Phys. Rev. B 52, 2843 (1995).

${ }^{17}$ Y. Yaish, J.-Y. Park, S. Rosenblatt, V. Sazonova, M. Brink, and P. L. McEuen, Phys. Rev. Lett. 92, 046401 (2004).

${ }^{18}$ M. S. Purewal, B. H. Hong, A. Ravi, B. Chandra, J. Hone, and P. Kim, Phys. Rev. Lett. 98, 186808 (2007).

${ }^{19}$ M. Ahlskog, O. Herranen, A. Johansson, J. Leppäniemi, and D. Mtsuko, Phys. Rev. B 79, 155408 (2009).

${ }^{20}$ C. Schönenberger, A. Bachtold, C. Strunk, J.-P. Salvetat, and L. Forro, Appl. Phys. A 69, 283 (1999).

${ }^{21}$ R. Tarkiainen, M. Ahlskog, A. Zyuzin, P. Hakonen, and M. Paalanen, Phys. Rev. B 69, 033402 (2004).

${ }^{22}$ A. Kanda, K. Tsukagoshi, Y. Aoyagi, and Y. Ootuka, Phys. Rev. Lett. 92, 036801 (2004).

${ }^{23}$ B. Stojetz, C. Miko, L. Forro, and Ch. Strunk, Phys. Rev. Lett. 94, 186802 (2005).

${ }^{24}$ S. Lombardo, J. H. Stathis, B. P. Linder, K. L. Pey, F. Palumbo, and C. H. Tung, J. Appl. Phys. 98, 121301 (2005).
${ }^{25}$ W. F. Brinkman, R. C. Dynes, and J. M. Rowell, J. Appl. Phys. 41, 1915 (1970).

${ }^{26}$ CRC Handbook of Chemistry and Physics (CRC Press, Boca Raton, FL, 2008).

${ }^{27}$ M. Shiraishi and M. Ata, Carbon 39, 1913 (2001).

${ }^{28}$ K. Bradley, S.-H. Jhi, P. G. Collins, J. H. Hone, M. L. Cohen, S. G. Louie, and A. Zettl, Phys. Rev. Lett. 85, 4361 (2000).

${ }^{29}$ P. Finnie, Y. Homma, and J. Lefebvre, Phys. Rev. Lett. 94, 247401 (2005).

${ }^{30}$ S.-H. Jhi, S. G. Louie, and M. L. Cohen, Phys. Rev. Lett. 85, 1710 (2000).

${ }^{31}$ G. Robert, V. Derycke, M. F. Goffman, S. Lenfant, D. Vuillaume, and J.-P. Bourgoin, Appl. Phys. Lett. 93, 143117 (2008).

${ }^{32}$ A. Bachtold, P. Hadley, T. Nakanishi, and C. Dekker, Science 294, 1317 (2001).

${ }^{33}$ C. Klinke, J. B. Hannon, A. Afzali, and Ph. Avouris, Nano Lett. 6, 906 (2006).

${ }^{34}$ P. Stokes and S. I. Khondaker, Appl. Phys. Lett. 92, 262107 (2008).

${ }^{35}$ R. T. Weitz, U. Zschieschang, F. Effenberger, H. Klauk, M. Burghard, and K. Kern, Nano Lett. 7, 22 (2007).

${ }^{36}$ Th. Glatzel, M. Ch. Lux-Steiner, E. Strassburg, A. Boag, and Y. Rosenwaks, Scanning Probe Microscopy, Vol. 1, pp. 113-131 (Springer, New York, 2007); M. Nonnenmacher, M. P. O'Boyle, and H. K. Wickramasinghe, Appl. Phys. Lett. 58, 2921 (1991).

${ }^{37}$ X. Cui, M. Freitag, R. Martel, L. Brus, and P. Avouris, Nano Lett. 3, 783 (2003).

${ }^{38}$ G. Chen, T. M. Paronyan, E. M. Pigos, G. U. Sumanasekra, and A. R. Harutyunyan, Appl. Phys. Lett. 95, 123118 (2009).

${ }^{39}$ H. Ulbricht, G. Moos, and T. Hertel, Phys. Rev. B 66, 075404 (2002).

${ }^{40}$ L. Britnell, R. V. Gorbachev, R. Jalil, B. D. Belle, F. Schedin, A. Mishchenko, T. Georgiou, M. I. Katsnelson, L. Eaves, S. V. Morozov, N. M. R. Peres, J. Leist, A. K. Geim, K. S. Novoselov, and L. A. Ponomarenko, Science 335, 947 (2012). 